\title{
Metodologia de Modelagem Matemática Automática Aplicada a Um Veículo Individual Compacto (VIC)
}

\author{
Myagyh A. P. Miranda*. Nestor Roqueiro. * \\ Rodrigo De S. Vieira** \\ *Departamento de Automação e Sistemas - DAS \\ Universidade Federal de Santa Catarina - UFSC \\ CEP: 88040-900, Florianópolis, SC, Brasil \\ (e-mail:myagyh.a.p.miranda@posgrad.ufsc.br, nestor.roqueiro@ufsc.br). \\ **Departamento de Engenharia Mecânica - EMC \\ Universidade Federal de Santa Catarina - UFSC \\ CEP: 88040-900, Florianópolis, SC, Brasil \\ (e-mail:rodrigo.vieira@ufsc.br)
}

\begin{abstract}
It was always a challenge to design, through mathematical models, systems and data. Since antiquity, the human being seeks to describe mathematically experimental systems to help you understand them and thus solve issues related to them. The objective of the work reported in this paper is to develop a methodology to automatically generate the mathematical modeling of dynamic systems, whether simple or advanced, applied case study, to the Individual Compact Vehicle (ICV). We use Maplesoft's math-based modeling tool, MapleSim TM, built on the Maple symbolic computing system. Directly from the description of the block diagram, the analytical equations of the system are automatically generated and simplified with a powerful symbolic mechanism. Automatic generation of equations is used in a mathematical tool to analyze systems, this design process drastically reduces the complexity of modeling. It is expected that the results generated by this methodology can receive applications on top of the models, not only control projects, but also optimization, robustness analysis or any other type of analysis.
\end{abstract}

Resumo: Sempre foi um desafio projetar, através de modelos matemáticos, sistemas e dados. O ser humano procura descrever matematicamente sistemas experimentais para ajudá-lo a compreendê-los e, assim, resolver questões relacionadas a eles. O objetivo do trabalho relatado neste artigo é desenvolver uma metodologia para gerar automaticamente a modelagem matemática de sistemas dinâmicos, simples ou avançados, aplicados ao estudo de caso, para o Veículo Compacto Individual (VIC). Usamos a ferramenta de modelagem baseada em matemática da Maplesoft, MapleSim TM, construída no sistema de computação simbólica Maple ${ }^{\mathrm{TM}}$. Diretamente a partir da descrição do diagrama de blocos, as equações analíticas do sistema são geradas automaticamente e simplificadas com um poderoso mecanismo simbólico. A geração automática de equações é usada em uma ferramenta matemática para analisar sistemas. Esse processo de design reduz drasticamente a complexidade da modelagem. Espera-se que os resultados gerados por esta metodologia possam receber aplicações nos modelos, não apenas projetos de controle, mas também otimização, análise de robustez ou qualquer outro tipo de análise.

Keywords: Methodology, Dynamic systems, Mathematical models, Maplesim

Palavras-chaves: Metodologia, Sistemas Dinâmicos, Modelos matemáticos, MapleSim ${ }^{\mathrm{TM}}$.

\section{INTRODUÇÃO}

A modelagem de sistemas é de extrema importância para o desenvolvimento da ciência e tecnologia. Sempre foi um desafio conceber, por meio de modelos matemáticos, sistemas e dados observados. O ser humano busca descrever matematicamente sistemas experimentais para ajudá-lo a entendê-los e, assim, solucionar questões relacionadas a eles.

A modelagem matemática está envolvida no estudo de sistemas dinâmicos, constituindo estes um conjunto de objetos agrupados por alguma interação ou interdependência, de modo que existam relações de causa e efeito nos fenômenos que ocorrem com os elementos desse conjunto e que a resposta de seus objetos constituintes varie no tempo (Monteiro 2006). Sendo na maioria das vezes de interesse o estudo de projetos de sistema dinâmico multi-domínio, os quais o design convencional difere de circuitos eletrônicos, sistemas mecânicos e sistemas de energia fluída, em parte devido à necessidade de integrar vários tipos de comportamento de energia como parte do projeto básico(Seo et al. 2003).

Considerando que a modelagem matemática destes sistemas dinâmicos é apresentada por um conjunto de equações diferenciais que podem ser obtidas através do estudo e cálculo manual, utilizam-se as leis físicas que governam um sistema 
particular, como, por exemplo as leis de Kirchhoff dos sistemas elétricos, ou as leis de Newton dos sistemas mecânicos(Ogata 2014). Equações que representam a dinâmica do sistema com precisão ou, pelo menos, de forma bastante aceitável. A meta do trabalho relatado neste artigo é desenvolver uma metodologia que permita gerar automaticamente a modelagem matemática de sistemas dinâmicos, sejam eles simples ou avançados.

Ressalta-se que um modelo matemático não é único para um dado sistema, ele pode ser descrito de diferentes maneiras, portanto, pode haver diversos modelos matemáticos, dependendo da perspectiva que é analisada. É possível aprimorar a exatidão de um modelo matemático aumentando sua complexidade, logo em um sistema completo incluem-se centenas de equações para descrevê-lo. Com isso em mente usamos a ferramenta de modelagem baseada em matemática da Maplesoft, MapleSim ${ }^{\mathrm{TM}}$, construída sobre o sistema de computação simbólica Maple, para ajudar na obtenção automática do modelo matemático, aplicado a um estudo de caso proposto, um veículo individual compacto (VIC) com o intuído de realizar aplicações sobre o modelo obtido.

Em geral, na solução de um novo problema, considera-se desejável arquitetar primeiramente um modelo simples de modo a se adquirir informações básicas e gerais para a solução. Posteriormente, um modelo matemático mais complexo poderá ser então elaborado e utilizado para análise mais detalhada.

\section{REVISÃO BIBLIOGRÁFICA}

Para o conhecimento dos autores, há alguns trabalhos relacionados a obtenção de modelos matemáticos diferentes, utilizando a ferramenta computacional supracitada no uso de soluções de problemas e modelagens, como o de Assaid (2011) que visa utilizá-la para determinar a solução exata da equação de Poisson em uma junção de barreira de Schottky aplicando três diferentes abordagens. Mulero e Tian (2013) utilizaram o Maple ${ }^{\mathrm{TM}}$ em seu trabalho para desenvolver um algoritmo para derivar Differential Algebraic Equations (DAEs) e gerar novas equações de estados que sejam válidas para os fluidos HardSphere (HS) ou Hard-Disk (HD) que são comuns no campo da física, química e engenharia química.

Aazou, Assaid e Jadida (2009) aplicam a mesma ferramenta citada para estudar a solução analítica exata das equações características de uma célula solar. Por vez, Rat (2011) apresenta uma modelagem dinâmica analítica de um robô paralelo Triglide usando simulações numéricas através do Maple ${ }^{\mathrm{TM}}$ e do software Adams. Buscando acelerar os processos de projeto e prototipagem de veículos elétricos híbridos, Dao e Friebe (2012) utilizaram o Maple ${ }^{\mathrm{TM}}$ e MapleSim $^{\mathrm{TM}}$ por ser uma ferramenta de modelagem física de multi-domínio para obter equações matemáticas que governam a resposta do modelo em uma forma simbólica otimizada.

Gachadoit e Renaud (2012) apresentam melhor as vantagens deste tipo de ferramenta numérica e simulação, usando como exemplo a modelagem e o design de um sistema de suspensão ativa. Wright e Soroka (2012) realizaram a modelagem e a simulação de motor de passo piezoeléctrico linear, utilizando da mesma ferramenta, e em Ren (2016) ao desenvolver um modelo simplificado em 1-D de uma bancada experimental de um sistema de metralhadora. De uma maneira geral, os trabalhos realizados por alguns autores são formas de otimização de algum método de modelagem, ou então, uma aplicação de seus recursos em alguns casos específicos, para gerar os modelos dinâmicos ou obter suas equações. No entanto, não há um processo claro relacionado a obtenção automática de modelos matemáticos.

\section{MATERIAIS E MÉTODOS}

Nesta seção, serão detalhadas as funções de cada um dos blocos funcionais apresentados no fluxograma da metodologia da Figura 1, bem como as decisões de implementações e as ferramentas utilizadas no desenvolvimento.

\subsection{Metodologia de Modelagem Automática}

A metodologia aplicada foca em obter o modelo matemático utilizando a ferramenta computacional citada anteriormente, que auxilia na geração automática das equações matemáticas que governam a resposta do sistema desenvolvido, possibilitando o desenvolvedor do sistema um melhor desempenho, em termos de tempo e precisão na geração de resultados. Vale ressaltar que o usuário necessita de conhecimento técnico e teórico sobre o sistema a ser desenvolvido, para que os resultados gerados durante o processo possam ser condizentes com os domínios a que pertence o sistema.

Para a aplicação dessa metodologia deve ser definido: ou o objeto, ou sistemas convencionais ou sistema dinâmico multidomínio, para que possam ser desenvolvidos com Maple ${ }^{\mathrm{TM}} \mathrm{e}$ MapleSim $^{\mathrm{TM}}$ onde nada precisa ser feito ou computado manualmente (com papel e lápis). Cada etapa do processo será feita usando a ferramenta equipada em um computador, alguns toolboxes adicionais, templates e comandos de execução disponíveis.

É desejável ter o modelo, que será estruturado na ferramenta, desenvolvido em um ambiente CAD para obtenção das propriedades dos elementos constituintes do problema selecionado. Ao se diversificar as ferramentas trabalhadas no desenvolvimento do modelo, é possível aproximar o modelo matemático de um modelo real diante da precisão de dados obtidos e aplicados ao sistema.

Depois de realizar o projeto do modelo em diagrama de blocos com os componentes físicos fornecidos na biblioteca do MapleSim $^{\mathrm{TM}}$, sua parametrização e especificar suas condições iniciais como desejado, o mesmo deve ser executado para que a simulação do modelo verifique se o seu comportamento está correto. Caso contrário, o modelo pode ser modificado até corresponder às especificações. Durante este passo (simulação), o MapleSim ${ }^{\mathrm{TM}}$ gera e simplifica automaticamente as equações do modelo e as simula com os solucionadores algébricos-diferenciais do Maple ${ }^{\mathrm{TM}}$, processo esse importante, pois ao gerar a extração das equações no Maple ${ }^{\mathrm{TM}}$, será obtido exatamente o mesmo conjunto de equações, uma vez que já 
foram validadas na simulação o que garante que elas estejam corretas.

Um sistema físico é geralmente representado em DAEs, desta maneira este software utiliza solucionadores de equações diferenciais que empregam técnicas avançadas para resolver tanto Ordinary Differential Equations (ODEs), DAEs ou Equações diferenciais parciais (PDEs), além possuir uma caixa de ferramentas que permite diversas conversões destas equações, sendo do interesse neste trabalhar com modelos em espaço de estados para realizar análises nos modelos obtidos.
Desta forma, ao obter os modelos lineares basta utilizar o comando de conversão do sistema que gera o modelo em espaço de estados do sistema, caso contrário existe uma limitação onde é necessário aplicar a linearização no modelo não linear, para então realizar a transformação do sistema em espaço de estados. Neste ponto ocasiona de ocorre a criação de estados "virtuais" incontroláveis e/ou não observáveis, dependendo da complexidade do modelo. Então é aplicada uma redução do sistema para remover esses estados.

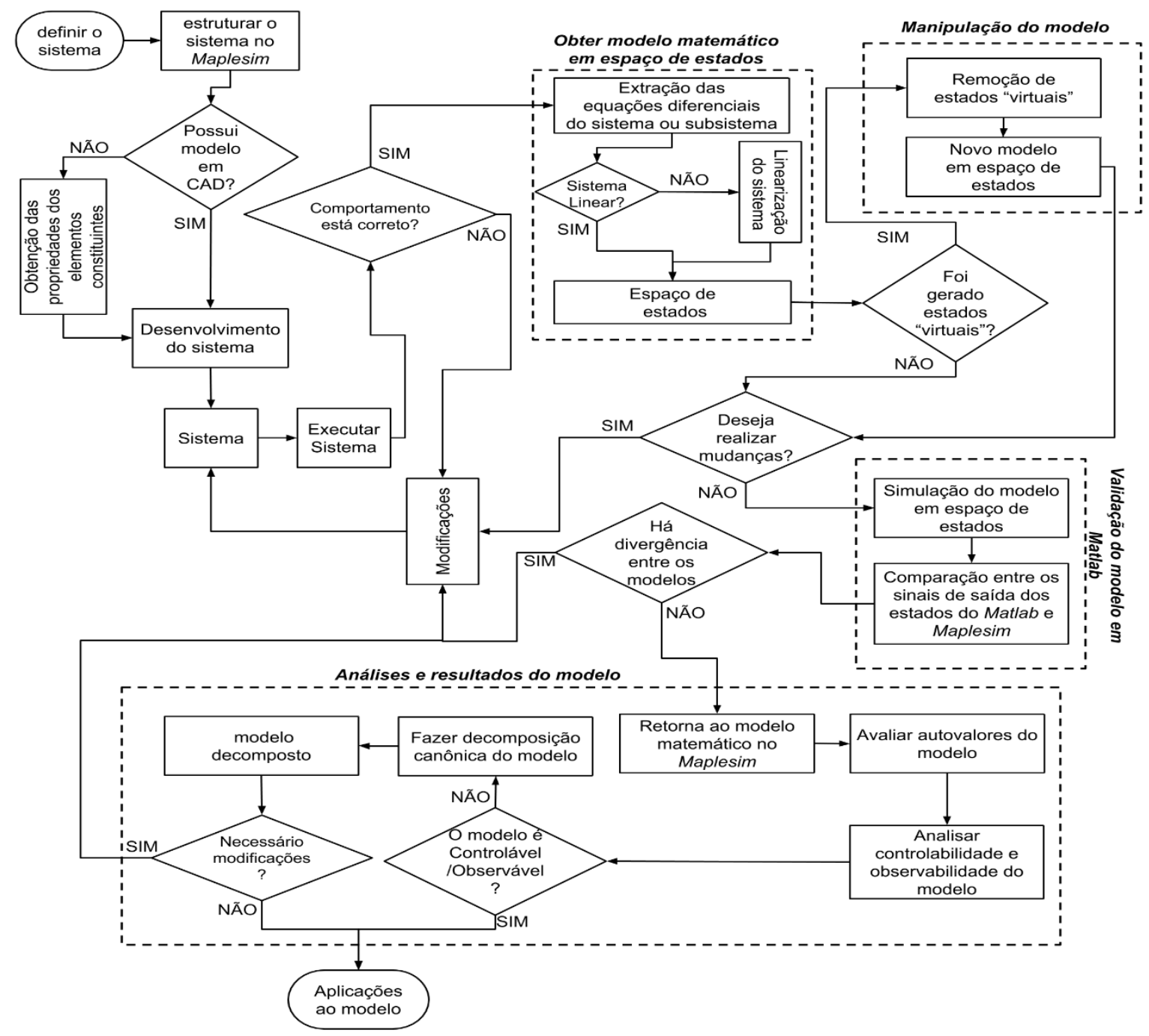

Figura 1: Fluxograma da metodologia de obtenção de modelos matemáticos automatizado com o auxílio da ferramenta Maple ${ }^{\mathrm{TM}}$ e MapleSim ${ }^{\mathrm{TM}}$.

Com o modelo em espaço de estados, parte para os pontos de análise e aplicações, primeiro avalia os autovalores do sistema, em seguida analisa a controlabilidade e observabilidade do mesmo, a fim de realizar aplicações de controle ao modelo. Em alguns casos o usuário obterá sistemas incontroláveis, neste ponto é preciso realizar uma decomposição canônica do sistema para obter as partes controláveis e incontroláveis separando esta parte para caso seja realizado um projeto de controle no sistema.
Obtido e analisado o modelo o usuário pode aplicar as estratégias de controle que desejar, seja no modelo matemático ou no modelo de simulação, bem como aplicar as demais transformações supracitadas no sistema, como por exemplo para funções de transferência, descretização do modelo ou até mesmo retorna o modelo para equações diferenciais.

Como forma de validação do modelo matemático obtido em espaço de estados, utiliza-se o software Matlab, aplicando o modelo em espaço de estado nele e comparando os sinais de saída dos estados desejados do sistema com os sinais obtidos 
pelo projeto desenvolvido em MapleSim ${ }^{\mathrm{TM}}$ o qual deu origem ao modelo. Assim, validando o modelo matemático do sistema, caso haja divergência deve ser feita alterações no modelo e em suas condições iniciais e replicar os passos.

A viabilidade da aplicação desta metodologia foi analisada empregando-a na obtenção de um modelo matemático de um Veículo Individual Compacto (VIC) e realizando aplicações em cima do modelo obtido, os dados obtidos serão apresentados e discutidos na próxima seção.

\section{VEÍCULO INDIVIDUAL COMPACTO (VIC)}

Nesta sessão vamos apresentar o desenvolvimento do sistema do VIC baseado na metodologia apresentada, a Figura 2(a) mostra o projeto conceitual do veículo. O modelo do sistema pode ser descrito como um pendulo invertido com duas rodas, sendo o modelo baseado no trabalho desenvolvido por Serafim, Roqueiro e Braga (2018), como mostra Figura 2(b).

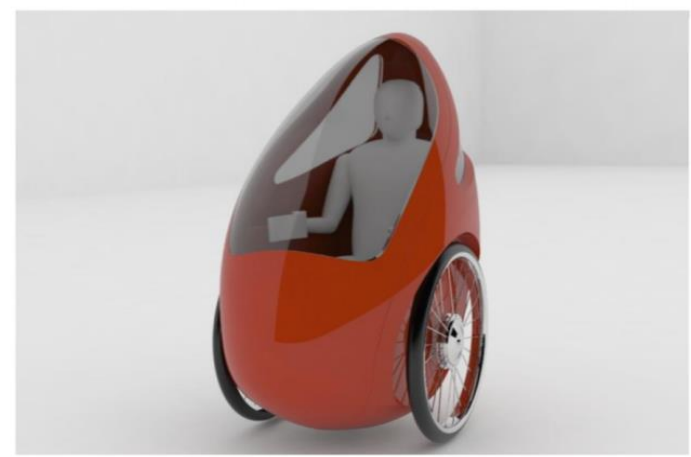

(a)

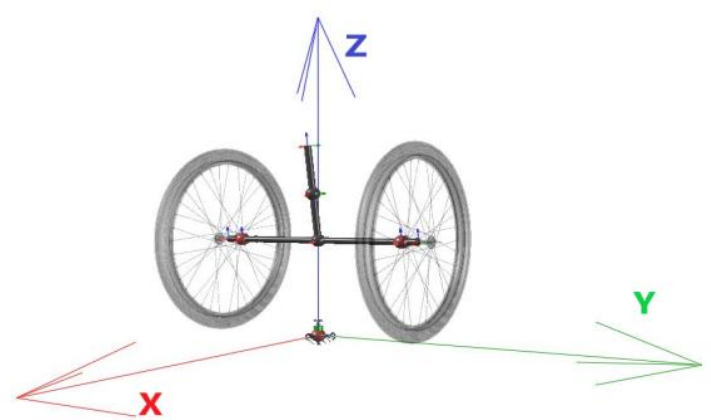

(b)

Figura 2: Representações do veículo: (a) Projeto conceitual do VIC. (b) Modelo baseado em um pêndulo invertido com duas rodas.

O modelo do veículo foi implementado na plataforma do MapleSim $^{\mathrm{TM}}$ (Figura 3), no qual as propriedades dos elementos constituintes deste modelo foram extraídas do modelo CAD, além de aplicar ao modelo condições iniciais e restrições necessárias à execução de sua simulação em malha aberta.

Com o sistema implantado, a geração de equações é feita através do Maple ${ }^{\mathrm{TM}}$ a partir deste modelo, tendo o sistema 8 graus de liberdade e modelado usando 8 coordenadas generalizadas, entretanto as equações são muito complexas para serem mostradas neste artigo, até mesmo para serem analisadas visualmente pelo usuário, as quais estão disponíveis através do $Q R$ code presente no Apêndice A. Contudo, é possível identificar que se trata de um sistema não-linear, e portanto, deve-se fazer a linearização do sistema para ser obtido o modelo em espaço de estados.

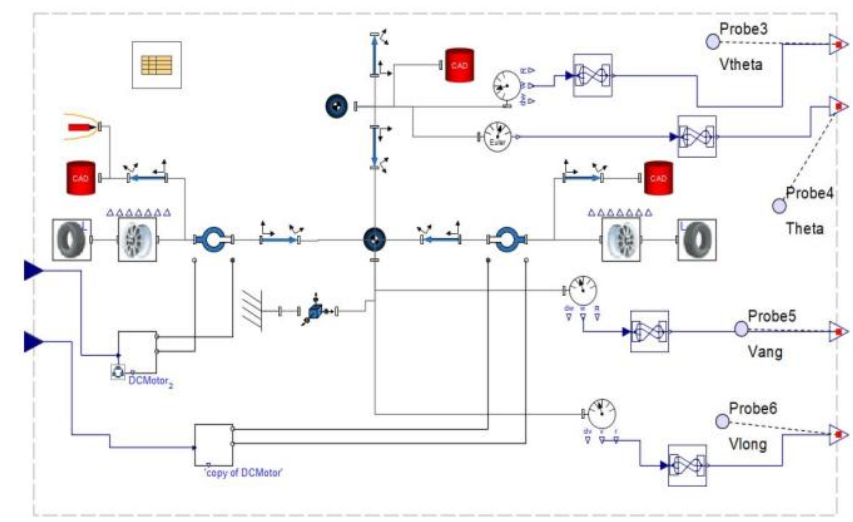

Figura 3: Modelo multi-corpos do VIC desenvolvido no MapleSim ${ }^{\text {TM }}$.

No processo de linearização foram observados dois pontos que são importantes ao se analisar a utilização deste processo. $\mathrm{O}$ primeiro está relacionado com o eixo de coordenadas, o qual é fixado entre as duas rodas. No MapleSim ${ }^{\mathrm{TM}}$ é aplicado uma restrição que desloca o eixo para o ponto desejado, entretanto ao realizar o processo da linearização o sistema não reconhece o eixo de coordenadas fixado entre as duas rodas do veículo, localizando as coordenadas como se estivessem na origem. Sendo assim, ajustou-se as condições iniciais fazendo um deslocamento de 0,05 metros em relação a coordenada $z$, retornando o eixo de coordenadas ao ponto desejado sem desestabilizar o sistema.

Outro ponto observado é com relação a geração de estados "virtuais". Após a linearização que resultou no modelo em espaço de estados com 22 estados, aplicou-se a remoção nestes estados "virtuais", gerando um modelo com 2 entradas, 4 saídas e 17 estados. Devido à complexidade, as matrizes de estados não foram incorporadas neste artigo, porém estão disponíveis para consulta por meio do $Q R$ code (Apêndice B), o qual também pode-se encontrar o modelo, bem como os estados. Como forma de validação, o modelo obtido foi aplicado ao Matlab que gerou sinais de saídas dos estados desejados. Assim, os sinais de ambos os sistemas (MapleSim ${ }^{\mathrm{TM}}$ e Matlab), em malha aberta, foram comparados como ilustra a Figura 4.

Por se tratar de um sistema de malha aberta, as simulações foram realizadas em um curto período de tempo para ser possível a observação do comportamento do sistema. Em virtude da linearização ser uma aproximação em torno de um ponto de operação, ela só pode levar à predição do comportamento do sistema em uma vizinhança deste ponto, podendo então observar que os sinais possuem o mesmo comportamento, havendo uma diferença em relação ao sistema não-linear, MapleSim ${ }^{\mathrm{TM}}$, e o sistema linearizado simulado via Matlab. 

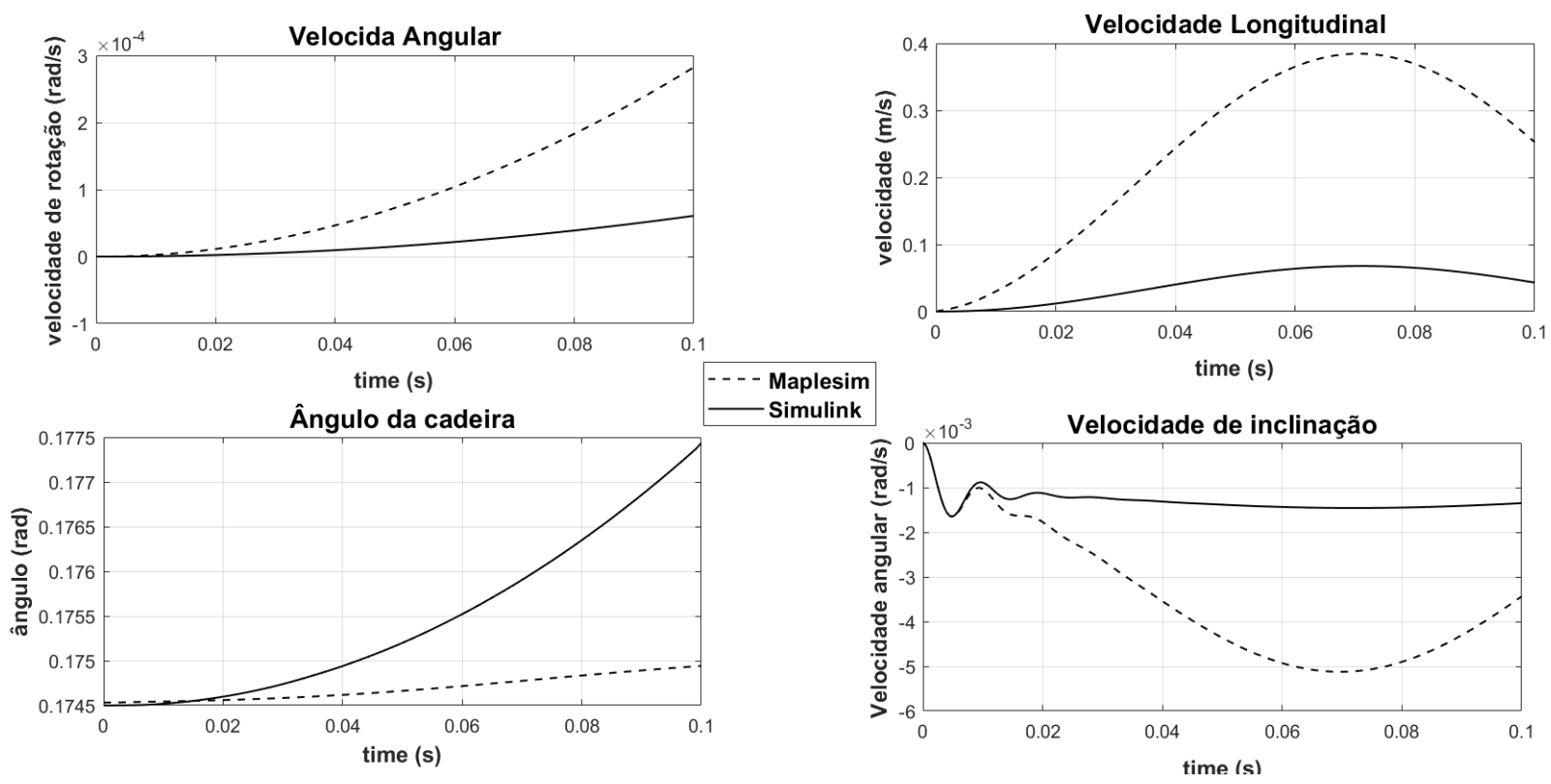

Figura 4: Sinais de saída dos sistemas no MapleSim ${ }^{\mathrm{TM}}$ e Matlab em malha aberta.

A partir deste ponto foi analisado os autovalores do modelo, em que constatou-se o estado instável que caracteriza o sistema de pendulo invertido, bem como a observabilidade do sistema, contudo o sistema demonstrou-se não ser controlável. Partindo para a etapa em que é realizada a decomposição canônica do sistema, parte em que é separada a parte controlável e não controlável. Com esta etapa é possível empregar um controlador para gerar a estabilidade no sistema, o modelo da parte controlável pode ser visto no $Q R$ code presente no Apêndice B.

\subsection{Controlador do VIC}

O método usado para projetar o controlador é clássico da teoria de controle. Trata-se do projeto por alocação de polos para sistemas de espaço de estado. O sistema de controle pode ser visto na Figura 5.

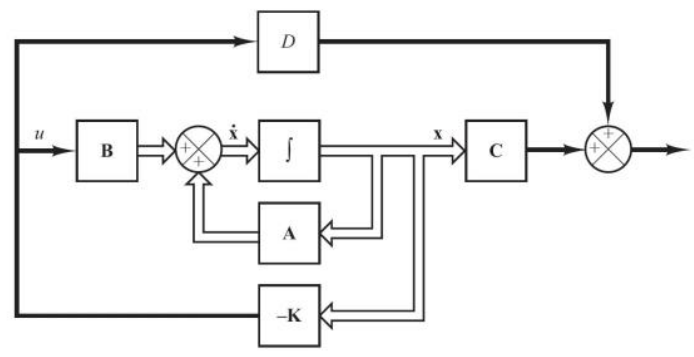

Figura 5: Sistema de controle em malha fechada.

Conforme a estrutura apresentada na Figura 5, o projeto do controlador linear foi realizado baseado no modelo controlável obtido na decomposição canônica, utilizando a técnica de alocação de pólos, e considerando os seguintes pólos desejáveis em malha fechada:

$$
p=[-50.13-50.1-50.02-50-49-7-5-3.13-3.01-3]
$$

Os ganhos encontrados foram:

$$
\begin{aligned}
& k=\left[\begin{array}{cccc}
-64,1128 & 11,5308 & -8,1085 & -11,3360 \\
-35,8973 & -12,9610 & 6,2613 & -8,30909 \\
90,0350 & -38,7231 & -23,8240 & \\
22.9096 & 33,6678 & 23,8324 \\
-23,5517 & -0,0388 & 0,2441 \\
-23,6964 & 0,2631 & -0,0219
\end{array}\right] .
\end{aligned}
$$

\subsection{Resultados via simulação}

O projeto de controle visto anteriormente, foi aplicado no modelo não linear, desenvolvido no MapleSim ${ }^{\mathrm{TM}}$. Este artigo apresenta a aplicação do controlador apoiado no modelo em tempo continuo, com o objetivo de verificar a dinâmica do sistema em malha fechada e a viabilidade da metodologia apresentada.

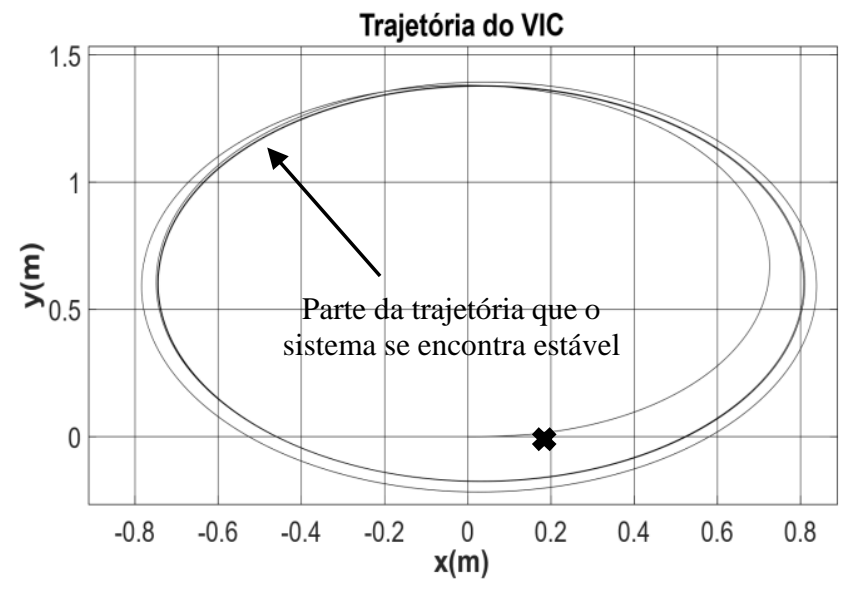

Figura 6: Trajetória percorrida via simulação. 
O trajeto que se inicia no ponto marcado conforme a Figura 6, foi simulado baseado no raio de curva mínimo que o veículo realiza. Logo, a parte apontada na Figura 6 apresenta o momento em que o VIC já atingiu a estabilidade, sendo que não são consideradas inclinações ou irregularidades no terreno.

Os resultados gerais das simulações são apresentados nas Figura 7 e Figura 8, mostrando a viabilidade da metodologia para realizar aplicações, como no caso aqui apresentado uma aplicação de controle sobre o modelo nos estados desejados a serem controlados, sendo o conjunto de gráficos:

$1^{\circ}$ - Ângulo de inclinação no sentido longitudinal do veículo;

$2^{\circ}$ - Velocidade de inclinação do veículo;

$3^{\circ}$ - Tensão nos motores;

$4^{\circ}$ - Velocidade longitudinal;

$5^{\circ}$ - Velocidade Angular.

\section{Ângulo de inclinação do VIC}

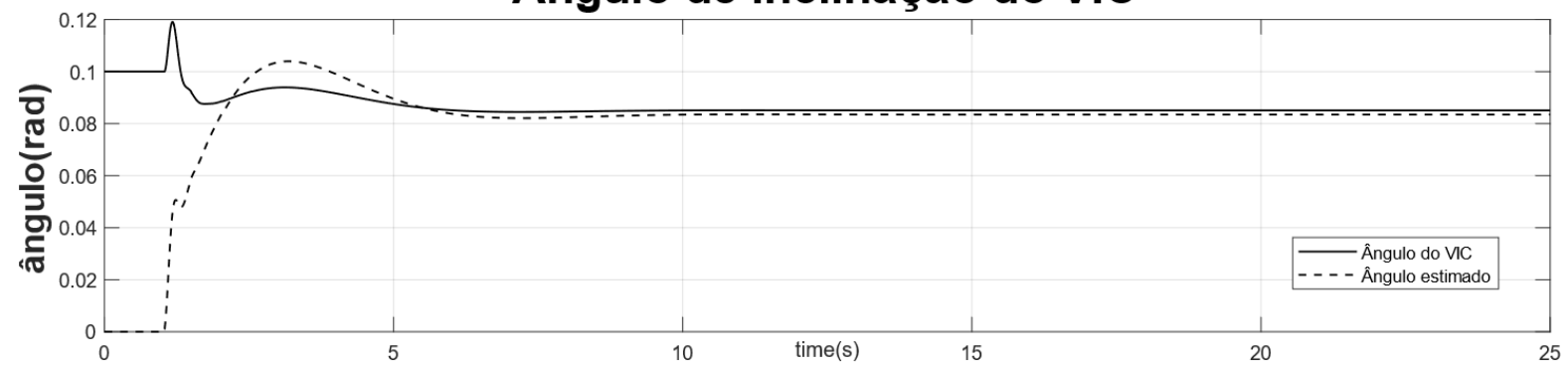

(a)

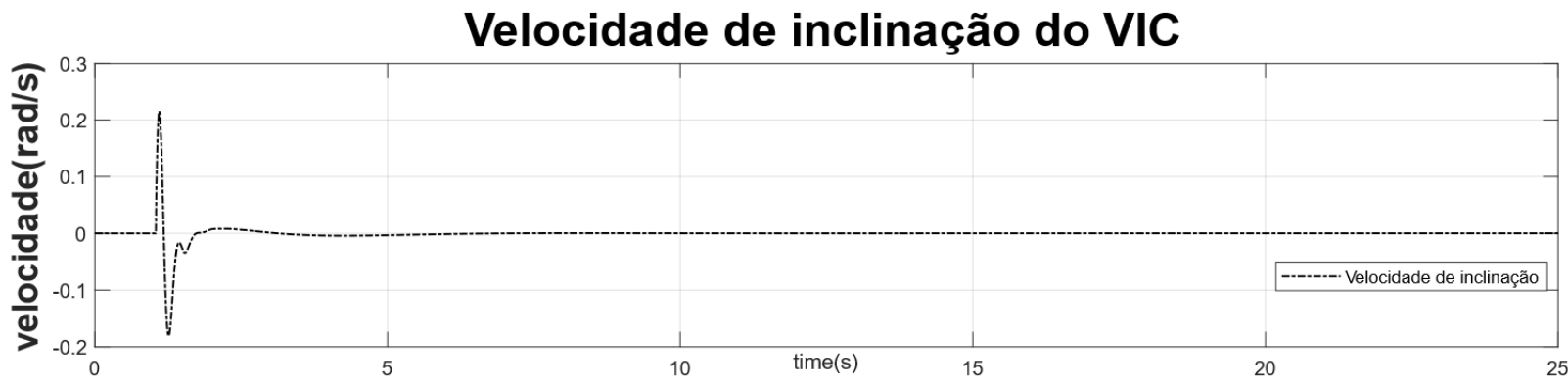

(b)

Figura 7: resultado do controle sobre: (a) o ângulo e (b) velocidade inclinação do VIC.

Como pode ser visto, o projeto de controle apresentou o resultado satisfatório. Observando a Figura 7(a), a curva tracejada representa o ângulo estimado de inclinação do veículo iniciada em $0 \mathrm{rad}$, e a curva em linha continua do ângulo do veículo simulado inicia em $0,1 \mathrm{rad}$ (esse fato é devido as condições iniciais impostas sobre modelo desenvolvido). Apesar da condição inicial, ambos os ângulos se estabilizam logo após os 5 segundos iniciais em aproximadamente $0,085 \mathrm{rad}$.

Outro fato que certifica o controle do ângulo do veículo pode ser observado na Figura 7(b), em que o período de oscilações presentes na velocidade de inclinação cessa no mesmo momento em que o ângulo é estabilizado.

Na Figura 8(a) as tensões máximas aplicadas aos motores em forma de degrau foram de $24 \mathrm{~V}$ no motor 1 e $10,59 \mathrm{~V}$ no motor 2 , possibilitando o VIC realizar o trajeto com curva de raio mínimo, a velocidade longitudinal máxima atingida neste caso foi de $0,8 \mathrm{~m}$ como pode ser visto na Figura $8(\mathrm{~b})$. Na Figura 8(c) a velocidade angular, ou seja, velocidade de rotação do veículo na realização de curvas foi de $2,14 \mathrm{rad} / \mathrm{s}$.

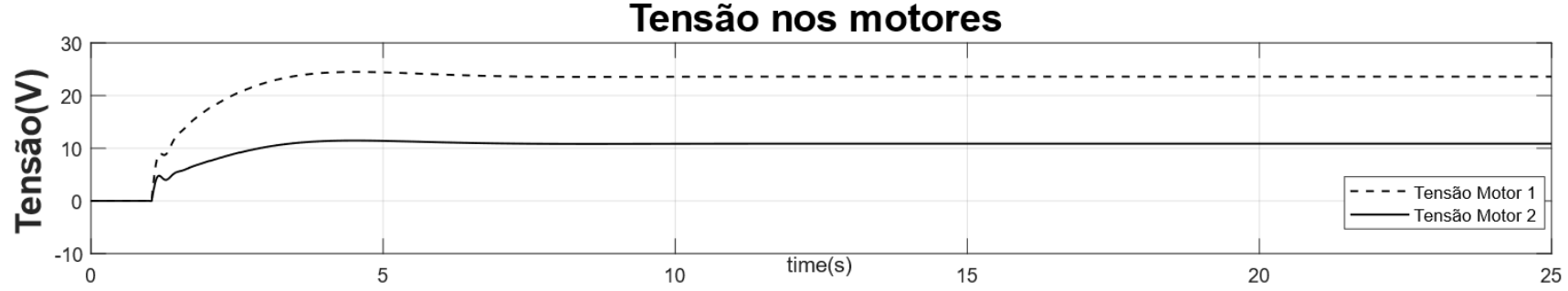

(a) 
Velocidade Longitudinal

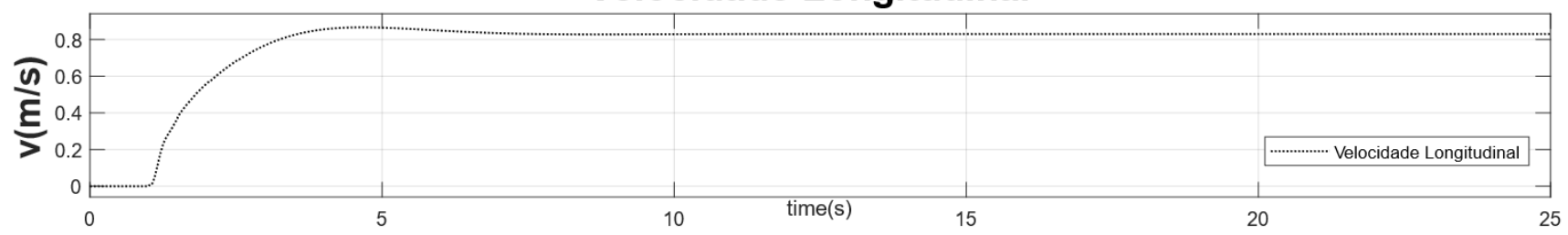

(b)

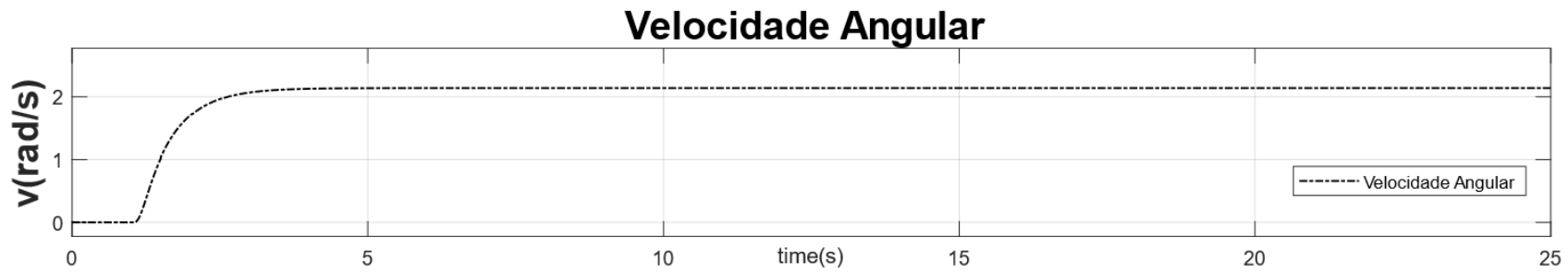

(c)

Figura 8: Resultados da simulação: (a) tensão nos motores, (b) velocidade longitudinal e (c) velocidade angular.

\section{CONCLUSÕES}

Neste trabalho, foi apresentado o desenvolvimento e aplicação de uma metodologia de geração automática de modelos matemáticos a partir de diagrama de blocos aplicando os benefícios oferecidos pelas ferramentas computacionais citas no trabalho. A metodologia desenvolvida foi aplicada ao veículo individual compacto permitindo uma alta aceleração do processo de desenvolvimento, sendo que nenhuma computação manual de equações foi necessária, o que é uma característica única.

A metodologia proposta é capaz de gerar um modelo dinâmico, admitindo aplicações como a de controle. O desenvolvimento do projeto de controle utilizado no sistema obteve respostas condizentes com o esperado, gerando uma contribuição tanto para o estudo de plataformas de manipulação móvel dinâmica de duas rodas, como pesquisas futuras para engenheiros, no desenvolvimento de estratégias de controle sofisticadas, assim como otimização, análise de robustez, entre outras aplicações.

\section{AGRADECIMENTOS}

"O presente trabalho foi realizado com apoio da Coordenação de Aperfeiçoamento de Pessoal de Nível Superior - Brasil (CAPES).

\section{REFERÊNCIAS}

Aazou, Safae, El Mahdi Assaid, and El Jadida. 2009. "MODELLING REAL PHOTOVOLTAIC SOLAR CELL USING MAPLE." (6): 394-97.

Assaid, El Mahdi, Safae Aazou, Asmaa Ibral, and El Jadida. 2011. "Finite Difference Numerical Solution of Poisson Equation in a Schottky Barrier Diode Using Maple." : 123-26.

Dao, Thanh-son, and Johannes Friebe. 2012. "Symbolic Techniques for Model-Based Design of Hybrid Electric Vehicles." : 1-10.

Gachadoit, N, and R Renaud. 2012. "Modeling and Design of

an Active Suspension System With Maple and Maplesim." Mecatronics-REM: 425-32.

Monteiro, L. H. A. 2006. Sistemas DinâMicos. São Paulo: Editora Livraria da Física.

Mulero, Ángel, and Jianxiang Tian. 2013. "Hard-Sphere Equations of State and Maple." Computing in Science \& Engineering: 56-62.

Ogata, Katsuhiko. 2014. Engenharia de Controle Moderno. $5^{\circ}$. Prentice Hall.

Rat, Nadia Ramona, Mircea Neagoe, Dorin Diaconescu, and Sergiu Dan Stan. 2011. "Dynamic Analysis of a Triglide Parallel Robot." : 245-49.

Ren, Haifeng. 2016. "Modeling and Simulating in MapleSim for a Bench Experimental System of Machine Gun." : 808-12.

Seo, Kisung et al. 2003. "Toward a Unified and Automated Design Methodology for Multi-Domain Dynamic Systems Using Bond Graphs and Genetic Programming." Mechatronics 13(8-9 SPEC.): 851-85.

Serafim, João Marcel, Nestor Roqueiro, and Rodrigo A. M. Braga. 2018. "Modelagam, Análise e Controle de Um Protótipo de Cadeira de Rodas Auto-Equilibrista." 13th IEEE International Conference on Industry Applications (INDUSCON): 9.

Wright, Derek, and Orysia Soroka. 2012. "Modeling and Simulation of a Linear Piezoelectric Stepper Motor in MapleSim." : 2746-49.

Apêndice A

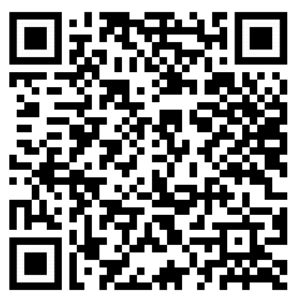

Apêndice B

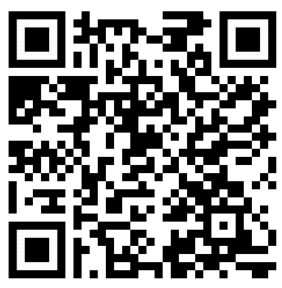

